\title{
Rationalization of Thermo-Mechanical Instabilities in Transient Additive Manufacturing of Ni-based Superalloys
}

Bryan $\operatorname{Lim}^{1 *}$, Xiang Yuan Cui ${ }^{1}$ and Simon Ringer ${ }^{1}$.

1. Australian Centre for Microscopy \& Microanalysis and School of Aerospace, Mechanical \& Mechatronic Engineering, The University of Sydney, Sydney, New South Wales, 2006, Australia.

* Corresponding Author: bryan.lim@sydney.edu.au

Recent discoveries have shown many parallels between metal additive manufacturing (AM) and physical metallurgy phenomena observed in casting, welding, powder metallurgy and thermo-mechanical processes $[1,2]$. However, recent studies have confirmed that the steady-state conditions assumed during traditional processes [3], are not valid in AM due to the formation of spatial and temporal transients, imposed by the abrupt, cyclical changes in energy delivery during the AM process [2,4-6]. For instance, the thermal gradients in Ti-6Al-4V were found to be in excess of $10^{6} \mathrm{~K} / \mathrm{m}$ [4]. Longer scan paths are believed to be responsible for the large thermal gradients and low frequencies of thermal gyrations [2,7]. This coupling of thermal gradients and thermal gyrations leads to interface velocities, as detected in a study of the Ni-based superalloy, Inconel-718 [8].

Hence, there is an intrinsic motivation to rationalize the effects of the new liquid/solid (1/s) and solid/solid $(\mathrm{s} / \mathrm{s})$ interface instabilities that arise with AM. Interfaces generated during AM will cause changes in local chemical bonding and associated physical properties, which will be investigated utilizing a combination of atom probe tomography (APT) and quantum mechanical modelling [9]. In particular, APT has recently been demonstrated to be uniquely powerful in the characterization of interfaces in materials [10-13], and allows for the gain into fundamental insights for interface engineering. This work will present new results from APT of Ni-based super alloys prepared by AM, for all major interface types involved, and across relevant combinations of the crystal types: FCC, BCC, $\mathrm{HCP}, \mathrm{L}_{2}$ and $\mathrm{B}_{2}$.

Furthermore, the principal atomistic modelling tools used in this investigation will be density functional theory (DFT) and molecular dynamics (MD). Computational simulations of atomic structure and microstructure will be used to rationalize the effects of dynamic thermal gradients on materials behavior. Model Ni-based interfaces with varying orientations and terminations will be investigated. The interfacial energies will be used as a basis to assess interface stability using DFT, and their behavior at finitetemperatures will be evaluated using MD [14]. 


\section{References:}

[1] Martin, J. H. et al., Nature 549 (2017), p. 365.

[2] Sames, W. J. et al., International Materials Reviews 61 (2016), p. 315.

[3] Christian, J. W. in "The Theory of Transformation in Metals and Alloys", (Pergamon Press, London) p.1.

[4] Thijs, L. et al., Acta Materialia 58 (2010), p. 3303.

[5] Verhaeghe, F. et al., Acta Materialia 57 (2009), p. 6006.

[6] Gu, D. D. et al., International Materials Reviews 57 (2012), p. 133.

[7] Kirka, M. M. et al., Scripta Materialia 135 (2017), p. 130.

[8] Dehoff, R. R. et al., Materials Science and Technology 31 (2015), p. 931.

[9] Cui, X.Y. and Ringer, S.P., Materials Characterization 146 (2018), p. 347.

[10] Ceguerra, A. V. et al., Current Opinion in Solid State and Materials Science 17 (2013), p. 224.

[11] Hono, K. et al., MRS Bulletin 41 (2016), p. 23.

[12] Liddicoat, P. V. et al., Nature Communications 1 (2010), p. 63.

[13] Yao, L. et al., Scripta Materialia 69 (2013), p. 622.

[14] The authors acknowledge the facilities and the scientific and technical assistance of Microscopy

Australia at the Australian Centre for Microscopy \& Microanalysis (ACMM) at the University of Sydney. They would also like to acknowledge the supercomputing resources provided by the National Computational Infrastructure (NCI) provided by the Australian Government, and the high-performance computing cluster Artemis provided by the University of Sydney. 